\title{
Two-dimensional magnetohydrodynamic turbulence in the small magnetic Prandtl number limit
}

\author{
David G. Dritschel ${ }^{1}$ and Steven M. Tobias ${ }^{2} \dagger$ \\ ${ }^{1}$ School of Mathematics and Statistics, University of St Andrews, St Andrews KY16 9SS, UK \\ 2 Department of Applied Mathematics, University of Leeds, Leeds LS2 9JT, UK
}

(Received 30 January 2012; revised 3 April 2012; accepted 16 April 2012; first published online 14 June 2012)

In this paper we introduce a new method for computations of two-dimensional magnetohydrodynamic (MHD) turbulence at low magnetic Prandtl number $P m=v / \eta$. When $P m \ll 1$, the magnetic field dissipates at a scale much larger than the velocity field. The method we utilize is a novel hybrid contour-spectral method, the "combined Lagrangian advection method', formally to integrate the equations with zero viscous dissipation. The method is compared with a standard pseudo-spectral method for decreasing $P m$ for the problem of decaying two-dimensional MHD turbulence. The method is shown to agree well for a wide range of imposed magnetic field strengths. Examples of problems for which such a method may prove invaluable are also given.

Key words: computational methods, MHD turbulence, turbulence simulation

\section{Introduction}

Modelling the dynamics of astrophysical and geophysical fluids is challenging owing to the vast range of length scales and time scales which need to be faithfully represented (e.g. Brandenburg \& Nordlund 2011). The conditions found in planetary and stellar interiors typically imply exceedingly small or large parameters in the governing equations, which in turn create difficulties for asymptotic analyses and numerical solutions. It is almost always the case, for instance, that astrophysical flows are characterized by very large fluid Reynolds numbers $R e=U L / v$ and are therefore turbulent.

A further complication for the numerical simulation of astrophysical flows is that the ionized plasma or liquid-metal flows are electrically conducting; moreover, the material properties of the fluid are such that the ratio of time scales for the diffusion of momentum relative to the magnetic field is very large. This disparity is often characterized by a magnetic Prandtl number $P m=v / \eta$ (the ratio of molecular viscosity to magnetic diffusivity) which is very small $\left(10^{-5}\right.$ in liquid metals and $10^{-5}-10^{-3}$ in stellar interiors). In the turbulent flows expected at high $R e$, this disparity leads to a large range of spatial scales between the dissipation scales of the kinetic and magnetic energies (see e.g. Schekochihin et al. 2005; Tobias \& Cattaneo 2008). In addition, astrophysical flows typically involve high $R m=P m R e$, and so both the velocity and magnetic field dissipate at scales much smaller than a typical energy input scale to

$\dagger$ Email address for correspondence: smt@amsta.leeds.ac.uk 
the turbulence. We stress again that resolving such a large range of scales remains a problem for all numerical procedures, in particular those attempting to construct turbulent dynamo action and to investigate the dynamics of magnetohydrodynamic turbulence at low Pm (Tobias, Cattaneo \& Boldyrev 2012).

It is against this backdrop that there has been an increased interest in the properties of stably stratified magnetohydrodynamic turbulence. Understanding the dynamics of the solar tachocline, a narrow stably stratified layer with strong latitudinal and radial differential rotation situated at the base of the solar convection zone, has become a central problem of solar and stellar physics (see e.g. Miesch 2005). This is because the tachocline is believed to play an important role in the solar dynamo (Tobias $\&$ Weiss 2007), the mixing of elements (see e.g. Gough 2007) and the processes that control the spin-down of the Sun. The turbulence within this layer is believed to play a crucial role in redistributing angular momentum and mixing and yet remains poorly understood (see e.g. Tobias 2005; Wood \& McIntyre 2011). Recent investigations using both direct numerical simulations and direct statistical simulations have demonstrated that magnetic fields can strongly affect the dynamics of stably stratified turbulent flows (Tobias, Diamond \& Hughes 2007; Tobias, Dagon \& Marston 2011). However, these investigations have necessarily been limited to moderate values of the magnetic Prandtl number Pm, and have often parameterized the dissipation of momentum via an ad hoc hyperviscosity.

In this paper we introduce a numerical method that is optimized for studying the dynamics of stably stratified magnetohydrodynamic flows at low Pm. This method involves implementing a contour-advection scheme (Dritschel \& Ambaum 1997) for the (scalar) vorticity evolution, that conserves the ideal invariants of the flow in the absence of a magnetic field, and that can also include non-conservative effects like that arising from the Lorentz force (Dritschel \& Fontane 2010). Importantly, this method allows one to study inviscid flows, with exceedingly small levels of numerical dissipation (Dritschel \& Scott 2009). In tandem the evolution of the magnetic field is computed accurately using pseudo-spectral methods, using sufficient magnetic diffusion to faithfully resolve magnetic dissipation. We test the accuracy and speed of this method against a pure pseudo-spectral method that is optimized for use on massively parallel architectures for the simple model problem of unforced twodimensional magnetohydrodynamic turbulence. We demonstrate that as the magnetic Prandtl number is decreased at fixed $R m$ then the results of the pure pseudo-spectral method tend to those of the contour-advection method. This gives us confidence that contour advection provides an accurate description of the small-Pm limit of magnetohydrodynamic turbulence.

The paper is organized as follows. In the next section, we introduce the model problem to be studied and describe the two numerical methods implemented. We follow this in $\S 3$ with a detailed comparison of the results produced by the two methods, before concluding with a discussion of the implications of our results for further investigations in $\S 4$.

\section{Problem formulation}

\subsection{Physical model}

We examine a simplified magnetohydrodynamical (MHD) model of the lower solar tachocline (around 0.7 of the solar radius) where both stratification and rotation are believed to be important, and where magnetic forces play a significant role (Tobias 2010). In this region, the ratio of the Coriolis frequency $f$ to buoyancy frequency $N$ 
tends to be small (Miesch 2005), resulting in layerwise two-dimensional (dominantly horizontal) fluid motion, analogous to that found in the Earth's atmosphere and oceans (Gill 1982; Hoskins, McIntyre \& Robertson 1985). A mean horizontal magnetic field further enhances two-dimensional motions (Tobias 2005). Here, therefore, we consider the simplest purely two-dimensional model as a starting point for understanding the competing effects of fluid turbulence and magnetism. Though this is not a limitation of the method, fluid motions are assumed slow enough to neglect compressibility, which is a good approximation for the tachocline where the Mach number of motions is believed to be of the order of $10^{-4}$ (see e.g. Ossendrijver 2003).

The two-dimensional motion is fully described by the scalar vertical vorticity $\omega$ together with a scalar potential $A$ representing the magnetic field

$$
\boldsymbol{B}=B_{0} \hat{\boldsymbol{e}}_{x}+\left(-A_{y}, A_{x}, 0\right)
$$

where $B_{0}$ is the constant mean magnetic field in the $x$-direction, and where subscripts $x$ and $y$ denote partial derivatives. Similarly, the incompressible fluid motion is represented by a streamfunction $\psi$,

$$
\boldsymbol{u}=\left(-\psi_{y}, \psi_{x}, 0\right) \text {. }
$$

The evolution equations for $\omega$ and $A$ follow from taking the vertical components of the vorticity and induction equations:

$$
\begin{gathered}
\omega_{t}+J(\psi, \omega)=J\left(A, \nabla^{2} A\right)+B_{0} \nabla^{2} A_{x}+v \nabla^{2} \omega, \\
A_{t}+J(\psi, A)=B_{0} \psi_{x}+\eta \nabla^{2} A,
\end{gathered}
$$

where $v$ is the fluid viscosity, $\eta$ is the magnetic diffusivity, and $J(f, g) \equiv f_{x} g_{y}-f_{y} g_{x}$ is the Jacobian operator. Note that when $\eta=0$ the second equation implies that the quantity $-B_{0} y+A$ is materially conserved. Advection, particularly by a turbulent velocity field, deforms and stretches material contours, thereby generating strong gradients in $A$. This in turn feeds back into vorticity through the (curl of the) Lorentz force, the terms involving the current density $j=\nabla^{2} A$. In general, this causes the vorticity $\omega$ to weaken, as rotational motion is suppressed by the tension in the magnetic field lines. In short, magnetism tends to suppress turbulence.

In the absence of dissipation $(v=\eta=0)$, these equations conserve total energy $E=(1 / 2) \iint\left(u^{2}+B^{2}\right) \mathrm{d} x \mathrm{~d} y$, mean-square potential $\mathscr{A}=\iint A^{2} \mathrm{~d} x \mathrm{~d} y$ and crosshelicity $\mathscr{H}_{c}=\iint \boldsymbol{u} \cdot \boldsymbol{B} \mathrm{d} x \mathrm{~d} y=\iint \nabla \psi \cdot \nabla A \mathrm{~d} x \mathrm{~d} y$. The presence of a magnetic field breaks material conservation of vorticity, a powerful constraint operating in purely hydrodynamical turbulence. Nonetheless, when the field is weak, we expect enstrophy $Z=\iint \omega^{2} \mathrm{~d} x \mathrm{~d} y$ to cascade preferentially to small scales, and energy to build up at large scales - the so-called 'dual cascade' of two-dimensional turbulence (Dritschel et $a$ l. $2008 b$ and references therein). Enstrophy reaches arbitrarily small scales exponentially fast, inevitably leading to decay, whereas energy remains conserved. This 'selective decay' occurs in a different sense when the magnetic field is strong: then energy cascades to small scales, ultimately to be dissipated, whilst $A$ builds up at large scales (Kraichnan 1965; Iroshnikov 1967), as in this case the energy is minimized subject to the constraint of conservation of mean-square potential (see e.g. Biskamp 2003). There is also the possibility of dynamical alignment where $\boldsymbol{u}$ and $\boldsymbol{B}$ become either parallel or anti-parallel (as observed in the simulations examined in the next section).

In the solar tachocline, magnetic diffusion $\left(\eta \nabla^{2} A\right)$ is not negligible, and is considerably stronger than viscous diffusion $\left(v \nabla^{2} \omega\right)$ (Ossendrijver 2003). Magnetic diffusion breaks field lines and weakens the tension working against rotation. 
Sufficiently strong turbulence can overwhelm the Lorentz force and lead to long-lived vortices as in purely hydrodynamic turbulence at ultra-high Reynolds numbers (see Dritschel et al. 2008a,b).

The importance of magnetism, then, depends not only on the strength of the turbulence and the background magnetic field, but also on the magnetic diffusivity. A useful dimensionless measure of the importance of magnetism is given by (see e.g. Diamond et al. 2007)

$$
\gamma=\frac{B_{0}}{\eta^{1 / 2} U_{0}}
$$

where $U_{0}$ is a characteristic fluid velocity (e.g. the initial root-mean-square (r.m.s.) value). This dimensionless number measures the relative importance of the small-scale magnetic field compared to the velocity in fully developed turbulent flow. In two-dimensional turbulence, large-scale magnetic field is wrapped up into small-scale structures, thereby amplifying the energy in the field so that $\left\langle B^{2}\right\rangle=R m B_{0}^{2}$ (Cattaneo \& Vainshtein 1991). Hence $\gamma$ provides an estimate for when magnetic effects should become important on the small scale; i.e. the field should become significant when $\gamma$ is of order unity. Below, we explore how this parameter controls the evolution of turbulence, both in the inviscid limit $v=0$ and at finite magnetic Prandtl numbers $P m=v / \eta$.

\subsection{Numerical methods and initial conditions}

A standard pseudo-spectral method (hereafter PSM) is used to solve (2.4) in a doubly periodic $(2 \pi \times 2 \pi)$ domain for non-zero $v$ and $\eta$ (Cattaneo \& Tobias 2005). In addition, a novel hybrid contour-spectral method, the 'combined Lagrangian advection method' (CLAM, see Dritschel \& Fontane (2010)), is used at $P m=0$, specifically $v=0$ (the inviscid limit; see Dritschel \& Scott 2009 for justification). In both methods, the grid resolution was chosen by trial and error to ensure adequate resolution of the diffusion terms (e.g. to avoid any signs of Gibbs fringing). In CLAM, where there is only magnetic diffusion, we took $\eta=8 k_{0} U_{0} / k_{m}^{2}$, where $k_{0}$ is a characteristic wavenumber of the initial flow (see below), and $k_{m}$ is the maximum wavenumber associated with the basic grid (equal to half the grid resolution $n_{h}$ ). Note that in CLAM the vorticity $\omega$ is represented primarily by contours, allowing resolution of features up to 16 times smaller than the grid size. The results below are obtained with $n_{h}=1024$. The non-advective 'source' terms in the vorticity equation arising from the Lorentz force are handled by evolving a residual vorticity field $\omega_{d}$ alongside the advected contour field $\omega_{a}$. The combined field $\omega=\omega_{a}+\omega_{d}$ is frequently re-contoured and re-initialized as $\omega_{a}$ to minimize any numerical diffusion of the source terms (Dritschel \& Fontane 2010). An adaptive fourth-order Runge-Kutta scheme is used for the time stepping. All numerical parameters are determined from the grid resolution $n_{h}$ as discussed in Fontane \& Dritschel (2009). One parameter which does not depend on $n_{h}$ is the contour interval $\Delta \omega$. Here, we have chosen $\Delta \omega=1.35257$ to ensure 60 contours spanning the initial range in $\omega$. The recommended minimum number is 40 contours.

In the other method used, PSM, a higher grid resolution is necessary to resolve the viscous term when $P m=v / \eta<1$. Here, we doubled the basic resolution of $n_{h}=1024$ for each quartering of $P m$ starting from $P m=1$. That is, the grid resolution was chosen to be $1024 \mathrm{Pm}^{-1 / 2}$. Below, we consider $P m=1,1 / 4,1 / 16$ and $1 / 64$ and examine how the results converge to the $P m=0$ results obtained with CLAM. For each $P m$, we consider seven values of the dimensionless strength of the magnetic field (2.5) from very weak to very strong: $\gamma=0.1,0.2,0.5,1,2,5$ and 10. 
(a)

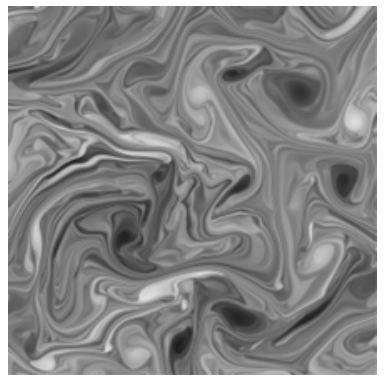

$(d)$

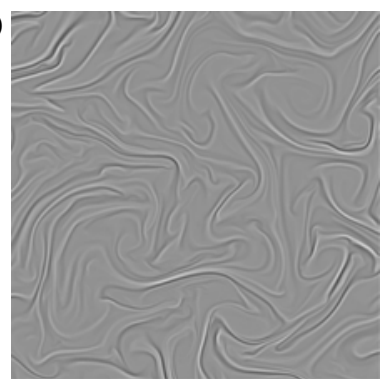

(b)

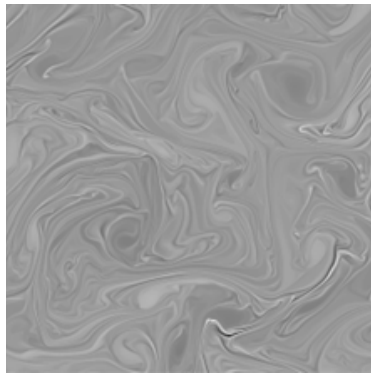

(e)

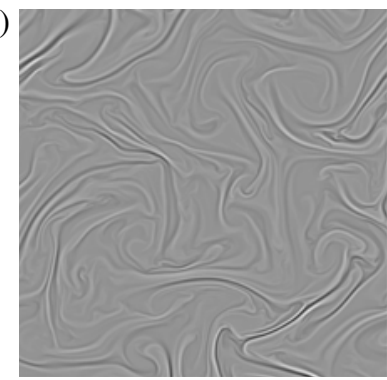

$(c)$

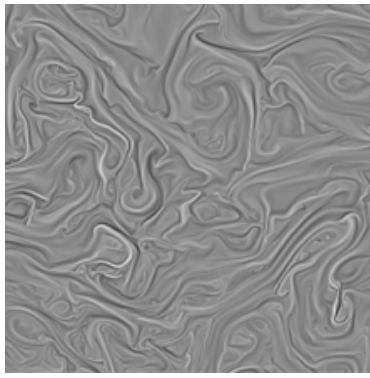

$(f)$

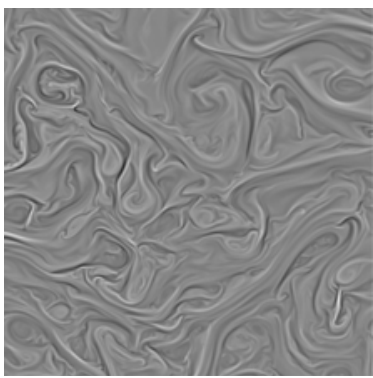

FIGURE 1. Final states of vorticity $\omega(a-c)$ and current density $j(d-f)$ for $P m=1 / 64$ and $\gamma=0.2(a, d), 1.0(b, e)$ and $10.0(c, f)$. Note we have zoomed in to show a quarter of the computational domain.

All simulations began from an identical initial flow state having the kinetic energy spectrum $\mathscr{E}=A k^{3} \mathrm{e}^{-2\left(k / k_{0}\right)^{2}}$ with $k_{0}=10$ and $A$ chosen so that the r.m.s. velocity $U_{0}=1$. Note that $\mathscr{E}(k)$ is maximum at $k=k_{0}$. The phases of individual Fourier modes are chosen at random (initially $|\omega|_{\max }=40.5771$ ). The initial perturbation magnetic potential $A$ is taken to be zero. In time, the twisting of magnetic field lines by the vorticity will generate $A$, and the objective here is to determine how the magnetic field interacts with the fluid turbulence as a function of the parameters $\gamma$ and Pm. Note that $\eta$ remains fixed in all simulations at $\eta=80 / 512^{2} \approx 3.05 \times 10^{-4}$.

\section{Results}

In this section, we compare the evolution of MHD turbulence as computed by the two numerical methods, PSM and CLAM. We contrast weak, moderate and strong magnetic fields $(\gamma=0.2,1$ and 10) and examine the convergence of the PSM solutions to the CLAM solutions as the magnetic Prandtl number $P m \rightarrow 0$.

\subsection{Flow characteristics and dependence on parameters}

We begin by describing the evolution of the PSM solutions for fixed resistivity $\eta=3.05176 \times 10^{-4}$ and initial conditions, but for varying field strength $\gamma$ and Pm. As noted above, because $P m=v / \eta$ decreases in proportion to the viscosity, smaller $\mathrm{Pm}$ requires higher grid resolution in PSM to faithfully resolve the dissipative length scale associated with vorticity. We have enforced this constraint in our simulations.

Figure 1 shows greyscale images of the vorticity and current density at the end of the simulations for three values of $\gamma(0.2,1.0$ and 10.0) for the lowest value of $\mathrm{Pm}$ considered. We note here that the high resolution of the simulations makes visualizing the fine structures on small length scales difficult and for this reason we only display 
a small patch of the turbulent flow and current. It is clear that for weak enough field $($ small $\gamma$ ) the vortices interact hydrodynamically and advect the passive magnetic field around. The current density is filamentary and lies predominantly at the edge of the vortex patches. This is a signature of kinematic flux expulsion (Weiss 1966) with the field being expelled by the eddies to the edge of cells. For passive magnetic field the turbulence acts so as to cascade the magnetic field down to the resistive scale, which for small $P m$ is at a much larger scale than the viscous scale. For intermediate field strengths $(\gamma=1)$ the evolution is broadly similar, except now the vorticity is significantly enhanced in regions near the current sheets. This tendency increases with $\gamma$, and for the strongest field strength considered the vorticity closely resembles the current density: $\omega \approx j$. At large $\gamma$, the magnetic field is clearly not passive and a significant modification of the vorticity field takes place. The solution has become largely 'Alfénized' (i.e. it takes the form of turbulent Alfvén waves - see e.g. Diamond et al. 2007). When $\eta=v=0$, it is well-known that (2.3) and (2.4) are exactly solved for $j=\omega$ (and $A=\psi$ ). Then, all nonlinear terms vanish, and the solution takes the general form $\psi=f\left(x-B_{0} t, y\right)$ for arbitrary $f$ (Biskamp \& Welter 1989). Remarkably, this solution appears to be an attractor for small $\eta$ and $v$ : arbitrary initial conditions tend to Alfvénize, at least before dissipation ultimately runs down the solution.

In the absence of forcing, the total energy (kinetic $\left\langle|\nabla \psi|^{2}\right\rangle / 2$ plus magnetic $\left.\left\langle|\nabla A|^{2}\right\rangle / 2\right)$ for all simulations decreases as a function of time. This implies that the r.m.s. velocity and hence the magnetic and fluid Reynolds numbers are functions of time. However $\mathrm{Pm}$ (which is independent of the flow amplitude) remains fixed throughout the simulations. The rate of decrease of the total energy is controlled by the dissipation - both magnetic and viscous - which are themselves functions of $\mathrm{Pm}$ and the typical length scales of the magnetic and vorticity fields. These typical length scales can be calculated using 'microscale' lengths defined as

$$
l_{u}=\frac{\left\langle|\nabla \psi|^{2}\right\rangle^{1 / 2}}{\left\langle\omega^{2}\right\rangle^{1 / 2}}, \quad l_{b}=\frac{\left\langle|\nabla A|^{2}\right\rangle^{1 / 2}}{\left\langle j^{2}\right\rangle^{1 / 2}},
$$

where the angled brackets indicate a domain average.

The evolution of these length scales as a function of time for two different $P m$ and three different imposed field strengths is shown in figure 2. Figure $2(a, b)$ shows the evolution of the fluid microscale $l_{u}$. It is clear that the evolution of this length scale is sensitive to the imposed field strength. For the relatively weak fields (the cases $\gamma=0.2$ and 1.0), the Lorentz force is weak and the evolution is dominantly hydrodynamic. The length scale starts to increase in both cases, which is a manifestation of the well-known inverse cascade to larger scales (Kraichnan 1967; Batchelor 1969). (The slightly faster growth for $P m=1$ is a diffusive effect.) Here the magnetic field is passive and so is shredded to the dissipative scale where ohmic diffusion acts. When the field is stronger $(\gamma=10)$ the Lorentz force is important, which has two primary consequences. The first (as demonstrated in figure $2 a, b$ ) is that, as vorticity is no longer materially conserved, the inverse cascade is halted and the fluid length scale actually decreases in time - a signature of a forward cascade, manifested in physical space by the formation of vortex sheets. The second is that the field can somewhat resist being shredded and so remains at a larger length scale (figure $2 c, d$ ). This picture can be seen to be relatively insensitive to $P m$ by comparing $(a, b)$ with $(c, d)$. The length scales grow slightly more rapidly at larger $P m$, simply because viscous diffusion is then larger. 


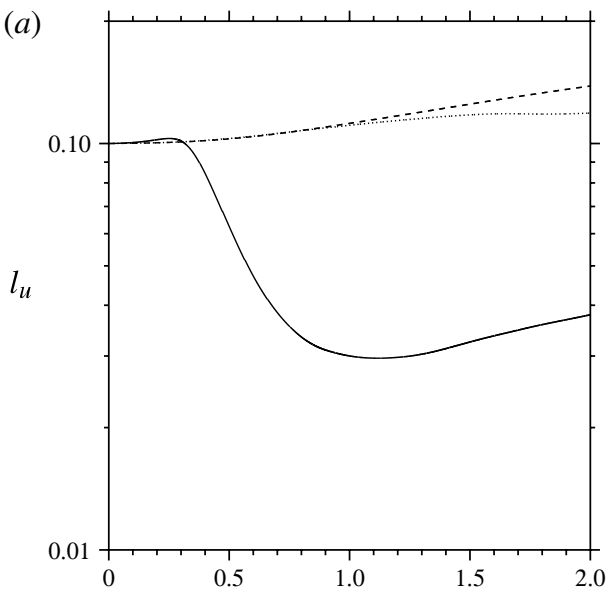

(b)

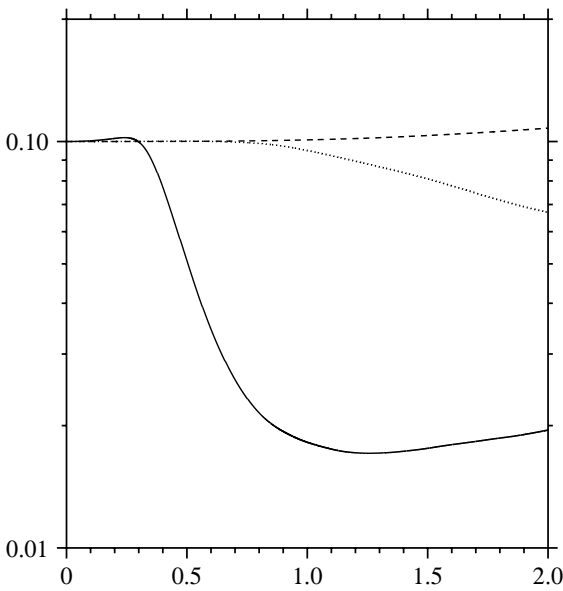

(c)

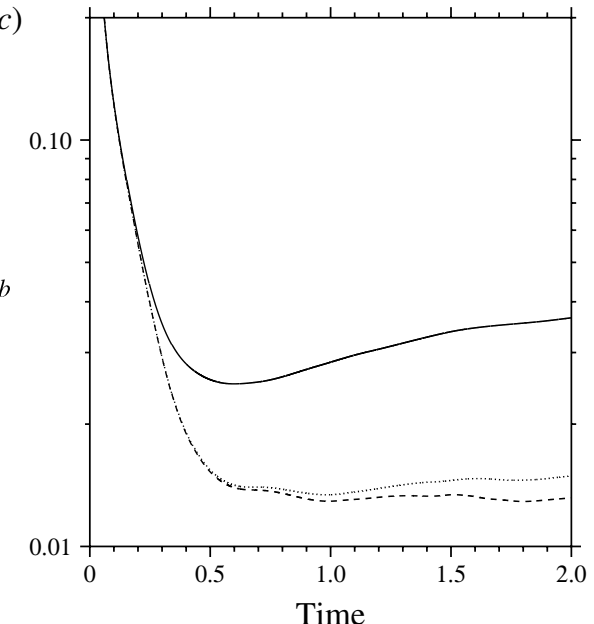

(d)

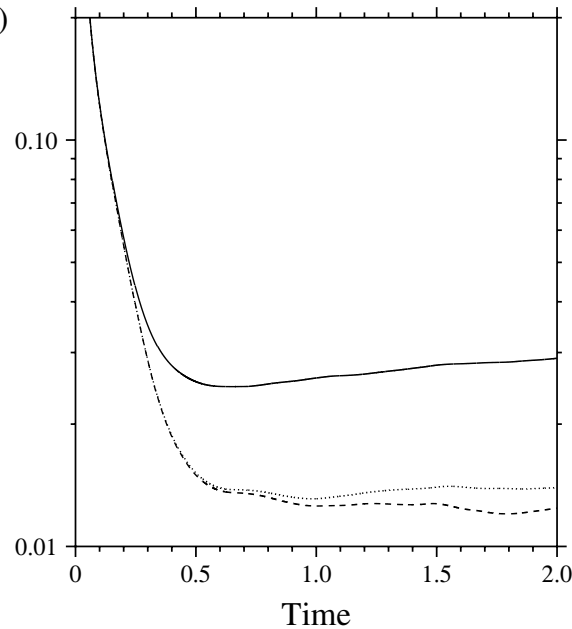

FIGURE 2. Evolution of the typical fluid and magnetic length scales as a function of time: $(a, b) l_{u}$ and $(c, d) l_{b}$, for $(a, c) P m=1$ and $(b, d) P m=1 / 64 ; \gamma=0.2$ (dashed), 1.0 (dotted) and 10.0 (solid).

Figures 3 and 4 show how the final state varies with magnetic Prandtl number $P m$, for weak and strong imposed magnetic fields respectively. In both cases, as $P m$ decreases the vorticity field exhibits filamentary structure on finer and finer scales, whilst the magnetic field exhibits current sheets which remain at a thickness limited by the magnetic resistivity. Notably, there appears to be a limiting form to the evolution as Pm approaches zero (also apparent in the evolution of the length scales in figure 2). It is this limiting behaviour that we are interested in. In the next subsection we compare the PSM simulations described here with formally inviscid CLAM simulations to determine whether CLAM can accurately model the $P m \rightarrow 0$ limit.

\subsection{Comparison with CLAM}

Having utilized PSM to obtain an understanding of the dynamics of the system as a function of imposed field strength and $\mathrm{Pm}$, we now examine how well CLAM captures 
(a)

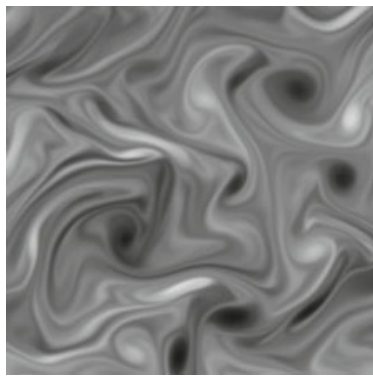

(d)

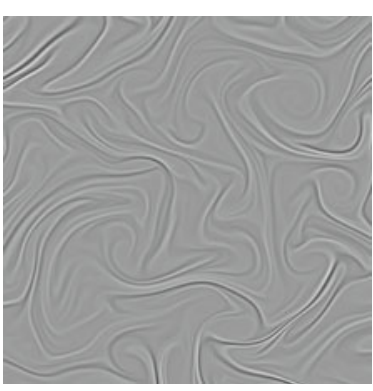

(b)

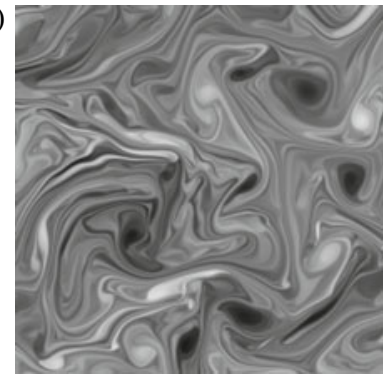

(c)

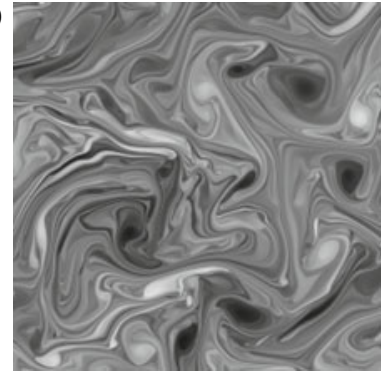

(e)
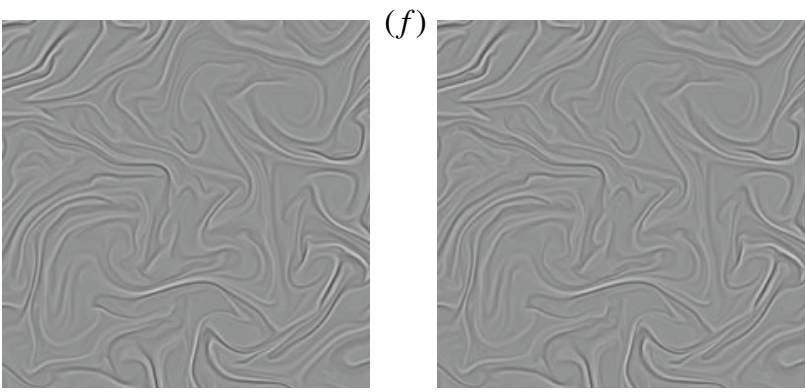

FIgURE 3. Final states of vorticity $\omega(a-c)$ and current density $j(d-f)$ for $\gamma=0.2$ and $P m=1(a, d), 1 / 16(b, e)$ and 1/64 $(c, f)$. Note that we have zoomed in to show a quarter of the computational domain.

(a)

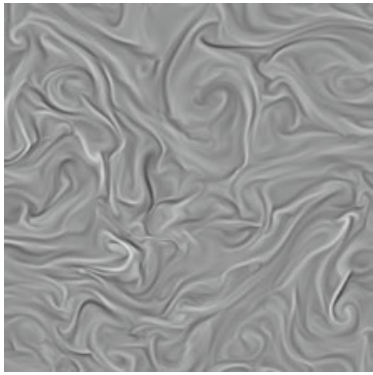

(d)

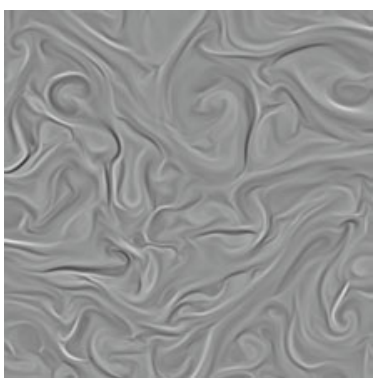

(b)

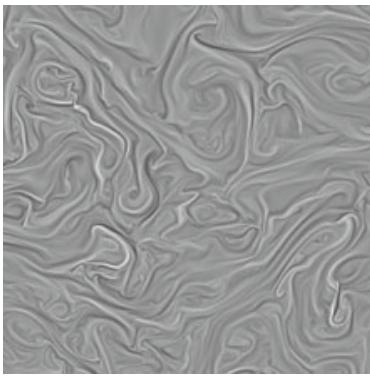

(e)

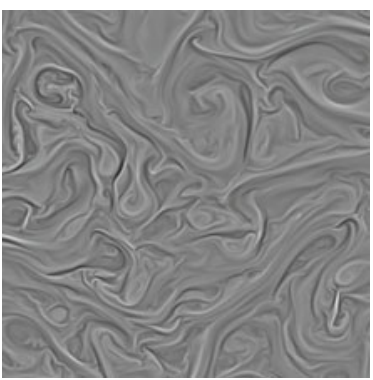

(c)

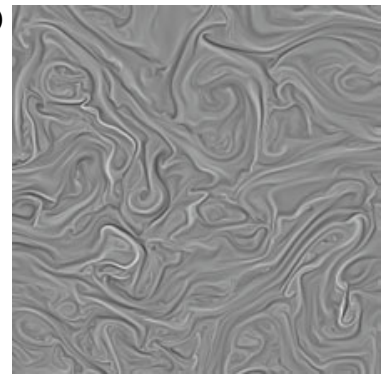

(f)

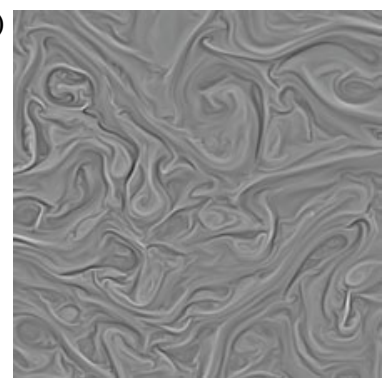

FIGURE 4. Final states of vorticity $\omega(a-c)$ and current density $j(d-f)$ for $\gamma=10$ and $P m=1(a, d), 1 / 16(b, e)$ and 1/64 $(c, f)$. Note we have zoomed in to show a quarter of the computational domain. 

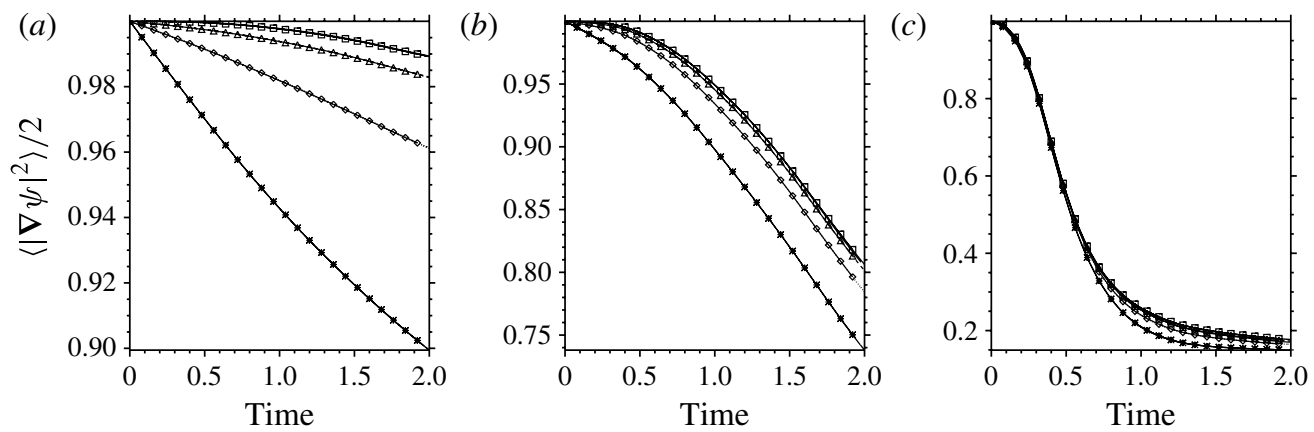

FIGURE 5. Time series of kinetic energy $\left\langle|\nabla \psi|^{2}\right\rangle / 2$ for $\gamma=0.2(a) 1.0(b)$ and 10.0 (c). Each plot shows the PSM results at magnetic Prandtl number $P m=1$ (asterisks) $P m=1 / 4$ (diamonds) $P m=1 / 16$ (triangles) and $P m=1 / 64$ (squares) are compared with the CLAM results (indistinguishable from the $P m=1 / 64$ results at this scale).
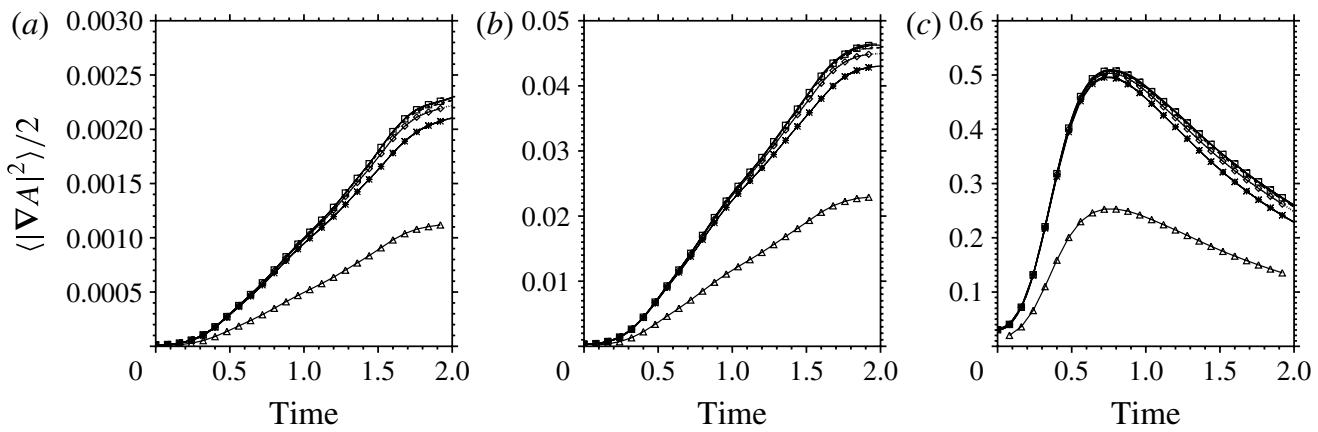

FIGURE 6. As figure 5 but magnetic energy $\left\langle|\nabla A|^{2}\right\rangle / 2$ is plotted.
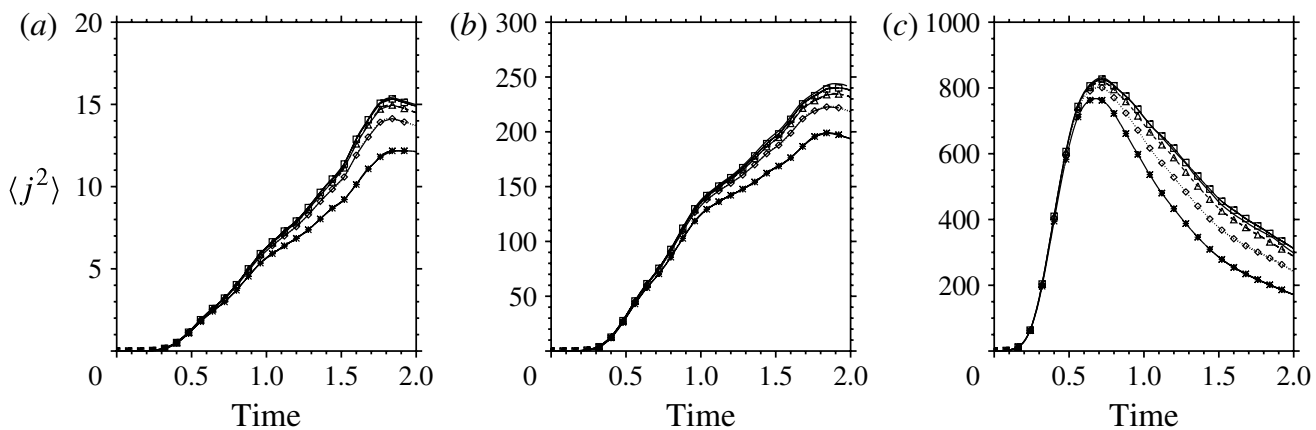

FIGURE 7. As figure 5 but mean-square current density $\left\langle j^{2}\right\rangle$ is plotted.

the behaviour of the $P m \rightarrow 0$ limit. To do this we compare the evolution and final state of the PSM simulations for various $\gamma$ and Pm with those of the formally inviscid CLAM simulation. Figures 5-7 show the evolution of the kinetic energy, magnetic energy and mean-square current density for these simulations. In each case as the 
system evolves kinetic energy is converted to magnetic energy and dissipation occurs via both ohmic and viscous mechanisms. For larger $\gamma$, the conversion to magnetic energy is more effective and the system quickly runs down via the more effective ohmic dissipation. The details of the evolution depend on Pm, which is most clearly seen in the evolution of the kinetic energy in the kinematic (weak magnetic field) case $(\gamma=0.2)$. For this weak field case, as $P m$ is decreased the dissipation is increasingly dominated by ohmic dissipation, which is itself small owing to the weakness of the field. However there is still some energy conversion to magnetic energy from kinetic and so the kinetic energy is reduced slightly over the course of the evolution. For stronger fields strong current sheets develop (see figure $7 c$ ) where the mean-square current density reaches large values and this leads to strong ohmic dissipation. In all cases the evolution is compared with that computed using CLAM for $P m=0$, shown by thick lines. What is immediately apparent is that CLAM provides an accurate representation of the limiting behaviour of the PSM simulations whether or not the evolution is one of inverse cascade to large scales (weak field) or of forward cascade to small scales (strong field). All results converge uniformly to the CLAM results as $P m \rightarrow 0$, irrespective of $\gamma$. This is quantified below.

A more detailed point-by-point comparison of the methods is given in figures 8 and 9. These figures compare the final states $(t=2)$ of vorticity and current density, respectively, from the PSM simulations for various $\gamma$ and for $P m=1 / 64$ with the corresponding final states of the CLAM simulations. The resemblance of the solutions for the two methods is striking for all values of $\gamma$ considered. CLAM reproduces fine details of the filamentary structure in both the vorticity and current density fields, where many individual features can be closely matched. Indeed pointwise correlations of the CLAM simulations with the pseudo-spectral simulations (for $P m=1 / 64$ ) reveal an extremely high degree of correlation (above 0.95 in most cases). These results demonstrate that CLAM can accurately and efficiently model the $P m \rightarrow 0$ limit.

Further evidence of the accuracy of CLAM can be seen in figures 10 and 11 . Figure 10 compares the spectrum for the kinetic energy of the PSM at Pm=1/64 with that of CLAM, for three values of $\gamma$. Although the shape of the energy spectrum changes considerably with the strength of the imposed magnetic field, CLAM gives an accurate representation of the finite-, but small- $\mathrm{Pm}$ limit. The only discrepancy occurs in the dissipative range and is exceptionally small. This discrepancy decreases as $P m \rightarrow 0$, as confirmed also by figure 11, which shows the difference of kinetic energy, magnetic energy and current density between the PSM solutions and the CLAM solutions (averaged over the period $1 \leqslant t \leqslant 2$ ) as a function of $P m$, again for three different values of imposed field $\gamma$. In all cases the error scales with $P m$, giving confidence that CLAM reproduces the correct behaviour in the $P m \rightarrow 0$ limit.

We conclude this section by noting that the CLAM simulations required $1.6 \mathrm{~h}$ on a single $3.2 \mathrm{GHz}$ Intel processor. The PSM simulations at $P m=1 / 64$ have a resolution of $8192 \times 8192$ and required $63 \mathrm{~h}$ on 128 processors. CLAM undoubtedly represents a cost-effective alternative to PSM for studying the behaviour of two-dimensional MHD turbulence at small $\mathrm{Pm}$.

\section{Conclusions}

In this paper we have examined the dependence of freely decaying two-dimensional magnetohydrodynamic turbulence on a non-dimensional measure of the magnetic field strength $\gamma$ and on the magnetic Prandtl number Pm. In particular, we have shown that the zero- $P m$ limit, i.e. an inviscid flow with finite magnetic resistivity, is a well-defined 
(a)

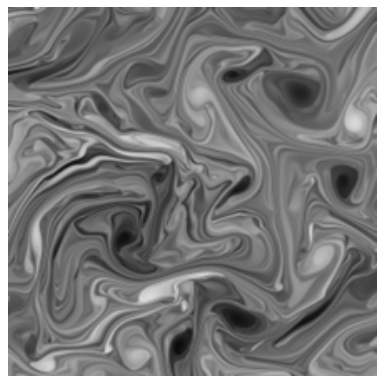

(d)

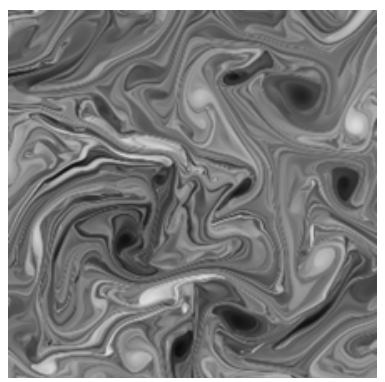

(b)

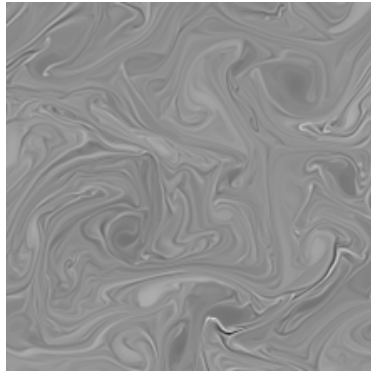

$(e)$

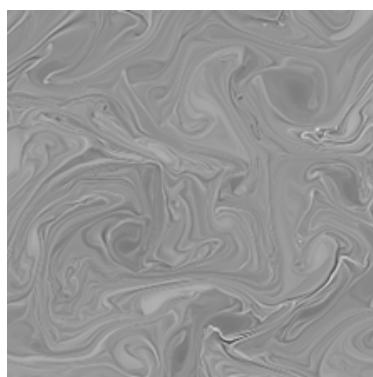

$(c)$

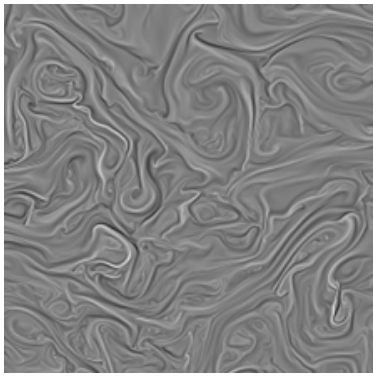

$(f)$

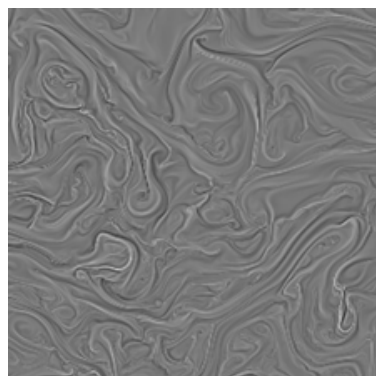

FIGURE 8. Final states of vorticity $\omega$ in the PSM simulations $(a-c)$ and in the CLAM simulations $(d-f)$ for $P m=1 / 64$ and $\gamma=0.2(a, d), 1.0(b, e)$ and $10.0(c, f)$. Note that we have zoomed in to show a quarter of the computational domain.

(a)

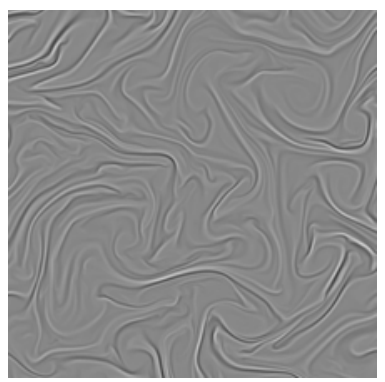

(d)

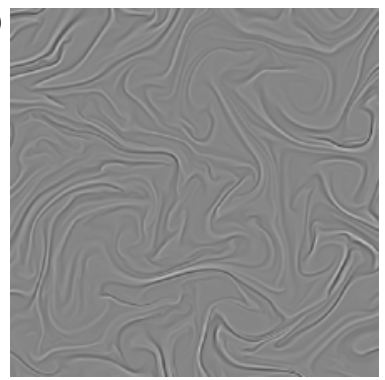

(b)

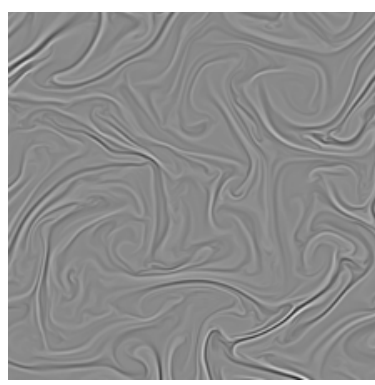

(e)

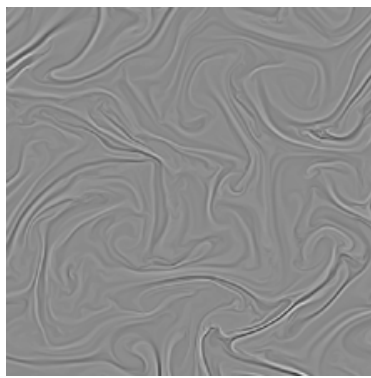

(c)

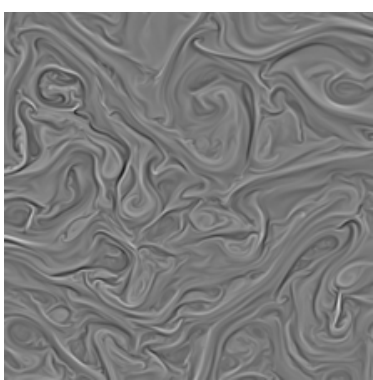

(f)

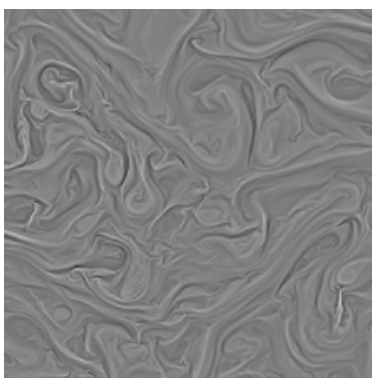

FIGURE 9. Final states of current density $j$ in the PSM simulations $(a-c)$ and in the CLAM simulations $(d-f)$ for $P m=1 / 64$ and $\gamma=0.2(a, d), 1.0(b, e)$ and $10.0(c, f)$. Note that we have zoomed in to show a quarter of the computational domain. 

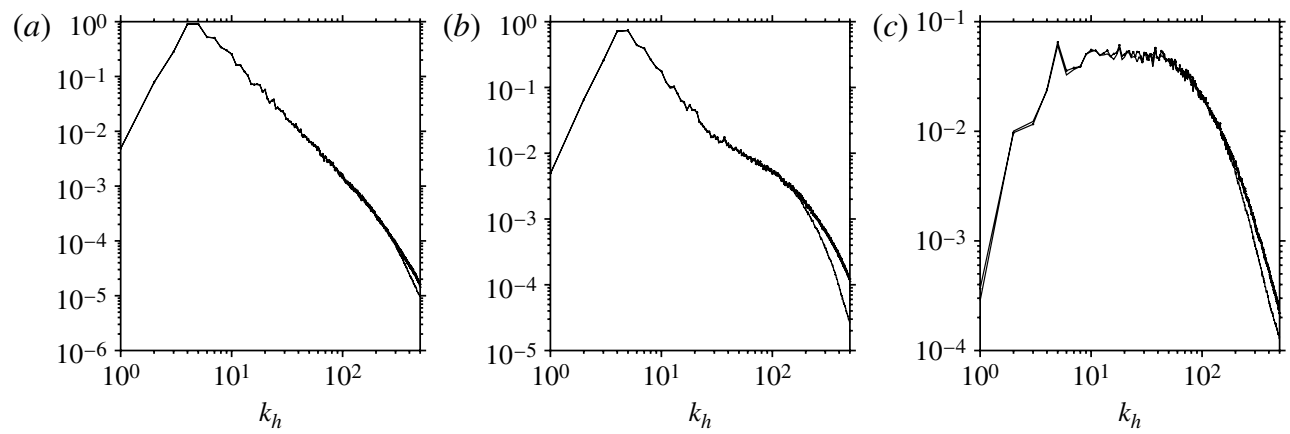

FIGURE 10. Kinetic energy spectra for CLAM (thin line) and PSM with $P m=1 / 64$ (thick line) for $\gamma=0.2(a) \gamma=1.0(b)$ and $\gamma=10.0(c)$. The spectra are taken at the end of the calculation.
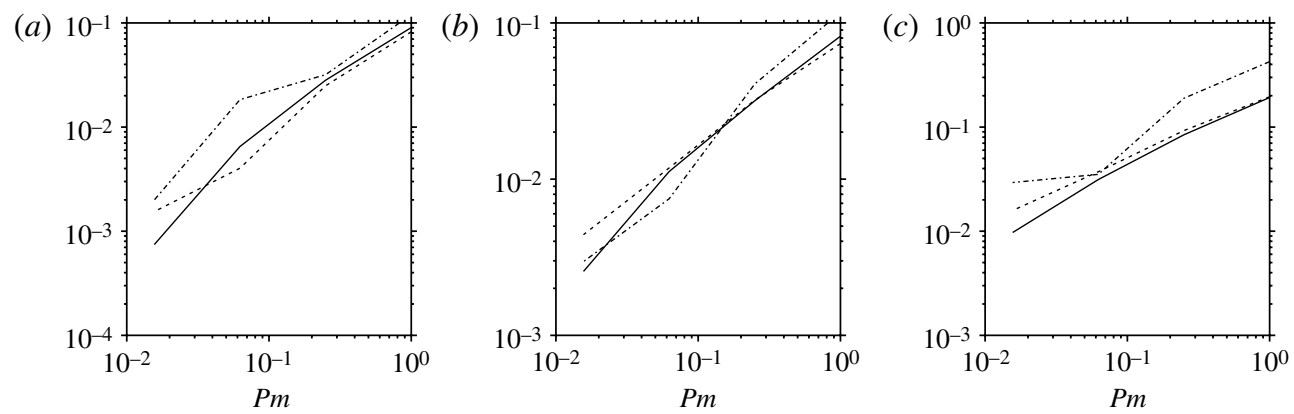

FIgURE 11. Comparison of PSM with CLAM as a function of Pm. Relative error (averaged over the period $1 \leqslant t \leqslant 2)$ in $(a)$ kinetic energy, $(b)$ magnetic energy and $(c)\left\langle j^{2}\right\rangle$ as a function of $P m$ for $\gamma=0.2$ (solid), $\gamma=1.0$ (dashed) and $\gamma=10$ (dot-dashed).

limit of the governing equations. Computationally, this limit is ideally suited to a novel hybrid contour-spectral method called CLAM (Dritschel \& Fontane 2010). This method permits one to study MHD turbulence on computational grids determined solely by the magnetic resistivity $\eta$. Explicit sub-grid modelling of the vorticity is provided by contour advection (Dritschel \& Ambaum 1997), thereby allowing huge gains in efficiency over widely used pseudo-spectral methods.

Given the power of CLAM demonstrated in this paper, it is of interest to discuss further applications. One important benefit of the method of computation in CLAM is that it allows the computation of the degree of breaking of Lagrangian potential vorticity (PV) conservation. For the two-dimensional 'barotropic' model considered in this paper, the potential vorticity is equivalent to the absolute vorticity $f+\omega$ (planetary plus relative), whilst in the more general shallow-water model (Gilman 2000) the potential vorticity is given by $q=(f+\omega) / h$, where $h$ is the local fluid depth. It is often argued that PV conservation plays an important role in determining the dynamics of rotating stratified systems (Dritschel \& Viúdez 2003; McKiver \& Dritschel 2008) and that the coupling of PV conservation to an inversion step plays an important role in for example jet formation and the generation of PV staircases (Dritschel \& McIntyre 2008). When magnetic fields are added both Lagrangian and 
total PV conservation are violated, via the Lorentz force term, and this can lead to the suppression of jets (Tobias et al. 2007). This study utilized the spectral code (PSM) for which it is difficult to measure the degree of breaking of Lagrangian PV conservation. We therefore propose to utilize CLAM to study the breaking of PV conservation by a magnetic field in a model of rotating shear instability on a $\beta$-plane. This is the simplest problem that can give an insight into the nonlinear behaviour of the magnetic field. As the jet undergoes instability, the potential vorticity is rearranged and mixed so as to reduce the destabilizing PV gradient. In the hydrodynamic case, as PV is a materially conserved quantity, this is an efficient process and saturation of the instability occurs rapidly. We shall quantify the role of the magnetic field, first of all in modifying the instability criterion, but more importantly in modifying PV conservation. Once the role of the magnetic field has been clarified, it will of course be of interest to extend the model to more realistic situations, involving the forcing of jets and to multi-layer shallow-water systems in more realistic geometries. The interaction between magnetic fields and fluid turbulence is a very rich topic, and its study will help us better understand the circumstances favouring the formation of coherent structures, which dominate energetically, and the fundamental mechanisms responsible.

\section{REFERENCES}

BATCHElOR, G. K. 1969 Computation of the energy spectrum in homogeneous two-dimensional turbulence. Phys. Fluids 12, 233-239.

Biskamp, D. 2003 Magnetohydrodynamic Turbulence. Cambridge University Press.

BiskAMP, D. \& Welter, H. 1989 Dynamics of decaying two-dimensional magnetohydrodynamic turbulence. Phys. Fluids B 1, 1964-1979.

Brandenburg, A. \& Nordlund, Å 2011 Astrophysical turbulence modelling. Rep. Prog. Phys. 74, 046901.

Cattaneo, F. \& Tobias, S. M. 2005 Interaction between dynamos at different scales. Phys. Fluids 17, 127105.

Cattaneo, F. \& Vainshtein, S. I. 1991 Suppression of turbulent transport by a weak magnetic field. Astrophys. J. 376, L21.

Diamond, P. H., Itoh, S.-I., Itoh, K. \& Silvers, L. J. $2007 \beta$-plane MHD turbulence and dissipation in the solar tachocline. In The Solar Tachocline (ed. D. W. Hughes, R. Rosner \& N. O. Weiss), p. 213. Cambridge University Press.

Dritschel, D. G. \& Ambaum, M. H. P. 1997 A contour-advective semi-Lagrangian numerical algorithm for simulating fine-scale conservative dynamical fields. Q. J. R. Meteorol. Soc. 123, $1097-1130$.

Dritschel, D. G. \& Fontane, J. 2010 The combined Lagrangian advection method. J. Comput. Phys. 229, 5408-5417.

Dritschel, D. G. \& MCIntyre, M. E. 2008 Multiple jets as PV staircases: the Phillips effect and the resilience of eddy-transport barriers. J. Atmos. Sci. 65, 855-874.

Dritschel, D. G. \& SCOTT, R. K. 2009 On the simulation of nearly inviscid two-dimensional turbulence. J. Comput. Phys. 228, 2707-2711.

Dritschel, D. G., Scott, R. K., Macaskill, C., Gottwald, G. A. \& Tran, C. V. $2008 a$ Unifying scaling theory for vortex dynamics in two-dimensional turbulence. Phys. Rev. Lett. 101 (9), 094501.

Dritschel, D. G., Scott, R. K., Macaskill, C., Gottwald, G. A. \& Tran, C. V. $2008 b$ Late time evolution of unforced inviscid two-dimensional turbulence. J. Fluid Mech. 640, 215-233.

DRITSChEL, D. G. \& ViúdeZ, Á. 2003 A balanced approach to modelling rotating stably stratified geophysical flows. J. Fluid Mech. 488, 123-150. 
Fontane, J. \& Dritschel, D. G. 2009 The HyperCASL algorithm: a new approach to the numerical simulation of geophysical flows. J. Comput. Phys. 228, 6411-6425.

Gill, A. E. 1982 Atmosphere-Ocean Dynamics. Academic.

Gilman, P. A. 2000 Magnetohydrodynamic 'Shallow Water' equations for the solar tachocline. Astrophys. J. 544, L79-82.

Gough, D. O. 2007 An introduction to the solar tachocline. In The Solar Tachocline (ed. D. W. Hughes, R. Rosner \& N. O. Weiss), p. 3 Cambridge University Press.

Hoskins, B. J., Mcintyre, M. E. \& Robertson, A. W. 1985 On the use and significance of isentropic potential-vorticity maps. Q. J. R. Meteorol. Soc. 111, 877-946.

IROSHNikOV, P. S. 1967 Turbulence of a conducting fluid in a strong magnetic field. Sov. Astron. 7, 566-571.

Kraichnan, R. H. 1965 Inertial-range spectrum of hydromagnetic turbulence. Phys. Fluids 8 , $1385-1387$.

Kraichnan, R. H. 1967 Inertial ranges in two-dimensional turbulence. Phys. Fluids 10, $1417-1423$.

McKiver, W. \& Dritschel, D. G. 2008 Balance in non-hydrostatic rotating stratified turbulence. J. Fluid Mech. 596, 201-219.

Miesch, M. S. 2005 Large-scale dynamics of the convection zone and tachocline. Living Rev. Solar Phys. 2, 1.

Ossendrijver, M. 2003 The solar dynamo. Astron. Astrophys. Rev. 11, 287-367.

Schekochihin, A. A., Haugen, N. E. L., Brandenburg, A., Cowley, S. C., Maron, J. L. \& MCWilliams, J. C. 2005 The onset of a small-scale turbulent dynamo at low magnetic Prandtl numbers. Astrophys. J. 625, L115-L118.

ToBiAs, S. M. 2005 The solar tachocline: formation, stability and its role in the solar dynamo. In Fluid Dynamics and Dynamos in Astrophysics and Geophysics (ed. A. M. Soward, C. A. Jones, D. W. Hughes \& N. O. Weiss), p. 193. CRC.

ToBIAs, S. M. 2010 The solar tachocline: a strudy in stably stratified MHD turbulence. In IUTAM Symposium on Turbulence in the Atmosphere and Oceans (ed. D. G. Dritschel), pp. 169-179. Springer.

Tobias, S. M. \& Cattaneo, F. 2008 Dynamo action in complex flows: the quick and the fast. J. Fluid Mech. 601, 101-122.

Tobias, S. M., Cattaneo, F. \& Boldyrev, S. 2012 MHD dynamos \& turbulence. In The Nature of Turbulence. Cambridge University Press.

Tobias, S. M., Dagon, K. \& Marston, J. B. 2011 Astrophysical fluid dynamics via direct statistical simulation. Astrophys. J. 727, 127.

Tobias, S. M., Diamond, P. H. \& Hughes, D. W. $2007 \beta$-plane magnetohydrodynamic turbulence in the solar tachocline. Astrophys. J. 667, L113-L116.

Tobias, S. \& Weiss, N. 2007 The solar dynamo and the tachocline. In The Solar Tachocline (ed. D. W. Hughes, R. Rosner \& N. O. Weiss), pp. 319-350. Cambridge University Press.

Weiss, N. O. 1966 The expulsion of magnetic flux by eddies. Proc. R. Soc. Lond. A 293, 310-328.

Wood, T. S. \& McIntyre, M. E. 2011 Polar confinement of the Sun's interior magnetic field by laminar magnetostrophic flow. J. Fluid Mech. 677, 445-482. 